\title{
Sampling and diversity of Escherichia coli from the enteric microbiota in patients with Escherichia coli bacteraemia
}

\author{
Mia Mosavie ${ }^{1}$, Oliver Blandy ${ }^{1}$, Elita Jauneikaite ${ }^{1}$, Isabel Caldas ${ }^{1}$, Matthew J. Ellington ${ }^{1,2}$, Neil Woodford ${ }^{1,2}$ \\ and Shiranee Sriskandan ${ }^{1 *}$ (D)
}

\begin{abstract}
Objective: The increase in Escherichia coli bloodstream infections mandates better characterisation of the relationship between commensal and invasive isolates. This study adopted a simple approach to characterize $E$. coli in the gut reservoir from patients with either E. coli or other Gram-negative bacteraemia, or those without bacteraemia, establishing strain collections suitable for genomic investigation. Enteric samples from patients in the three groups were cultured on selective chromogenic agar. Genetic diversity of prevailing E. coli strains in gut microbiota was estimated by RAPD-PCR.

Results: Enteric samples from E. coli bacteraemia patients yielded a median of one E. coli RAPD pattern (range 1-4) compared with two (range 1-5) from groups without E. coli bacteraemia. Of relevance to large-scale clinical studies, observed diversity of E. coli among hospitalised patients was not altered by sample type (rectal swab or stool), nor by increasing the colonies tested from 10 to 20 . Hospitalised patients demonstrated an apparently limited diversity of $E$. coli in the enteric microbiota and this was further reduced in those with $E$. coli bacteraemia. The reduced diversity of $E$. coli within the gut during $E$. coli bacteraemia raises the possibility that dominant strains may outcompete other lineages in patients with bloodstream infection.
\end{abstract}

Keywords: Escherichia coli, Microbiota, RAPD, Diversity, Bacteraemia, Stool, Rectal swab, Sepsis

\section{Introduction}

The increasing burden of Escherichia coli bacteraemia in the United Kingdom and dominance of antimicrobialresistant lineages worldwide [1-4] point to a need for better understanding of the reservoir and diversity of $E$. coli in the gut microbiota of those affected by invasive $E$. coli infections. E. coli is present in abundance in the adult gut microbiota, however the diversity of $E$. coli strains within the gut reservoir has only been studied in limited groups that do not include bacteraemia patients [5-10].

The enteric microbiota of such patients are hard to study, as bacteraemic patients will be acutely unwell, and

\footnotetext{
${ }^{*}$ Correspondence: s.sriskandan@imperial.ac.uk

${ }^{1}$ Health Protection Research Unit in Healthcare Associated Infections and Antimicrobial Resistance, Department of Medicine, Imperial College London, Hammersmith Campus, Du Cane Road, London W12 ONN, UK Full list of author information is available at the end of the article
}

stool samples are usually not readily available or clinically indicated, albeit that routinely-collected samples could represent an accessible sample source. Patients with suspected bacteraemia will usually receive empirical antimicrobial therapy within an hour of admission to hospital, consistent with international guidance on sepsis [11], thus all enteric samples are potentially antibiotic-exposed and may also be refrigerated for long periods of time before reaching the diagnostic laboratory, in turn potentially affecting microbiota [12].

Our aims were to establish methods to adequately sample E. coli strains present within the intestinal microbiota of hospital patients with E. coli bacteraemia; to evaluate control hospitalised groups; to assess scalability; and establish a sample collection for future large-scale genomic studies. Samples would need to be representative of the prevailing $E$. coli strains present in each patient 
without necessarily providing exhaustive insight into rare variants. The ability to detect $E$. coli variants in the enteric microbiota is related only to the underlying prevalence of such variants and how many colonies are counted [13]; to have a $90 \%$ likelihood of detecting a strain present in $20 \%$ of colonies, one needs to sample 11 colonies, while for a strain so rare it is present in just $5 \%$ of colonies, one needs to sample 45 colonies. Randomly Amplified Polymorphic DNA (RAPD) analysis can provide a relatively crude, but cheap, molecular method to differentiate strains based on amplicon banding patterns $[8,14,15]$ and was used to inform sampling strategy and characterise the samples obtained.

\section{Main text}

\section{Methods}

\section{Patients and samples}

Enteric samples were stool or rectal swabs from hospital in-patients obtained within $72 \mathrm{~h}$ of onset of Gram negative bacteraemia in west London National Health Service (NHS) Teaching Hospital sites between 1st July 2015 and 4th August 2016. Enteric samples were sought from patients prior to final microbiological identification thus were exposed to the same antimicrobial agents; groups were subsequently categorised into confirmed $E$. coli bacteraemia or other Gram negative non-E. coli bacteraemia. Controls were selected from inpatients within the same hospital wards that had not received an antibiotic in the preceding 30 days. Samples were obtained with informed consent except where rectal swabs had already been submitted to the hospital diagnostic laboratory for routine screening. In total, the study included 462 enteric samples (Additional file 1: Figure S1). Samples were refrigerated and analysed within $48 \mathrm{~h}$ unless otherwise stated, after any routine diagnostic testing had been completed. Preliminary studies demonstrated that refrigeration in the research lab for $48-72 \mathrm{~h}$ had little, or no, effect on the retrieval of E. coli from enteric samples though yield reduced by $20-25 \%$ over 10 days (data not shown). The study was approved by the Camden and Islington National Research Ethics Committee (Reference $14 / \mathrm{LO} / 2217$ ).

\section{Culture methods}

Approximately 10-60 mg of stool was suspended in $3 \mathrm{ml}$ $0.9 \%$ saline to achieve an optical density of $\mathrm{A}_{600} 0.1$ [16]. The suspension was diluted 100 -fold in $0.9 \%$ saline and $100 \mu \mathrm{l}(\sim 300-500$ colony forming units, CFU) cultured on coliform-selective chromogenic agar Brilliance $^{\text {TM }}$ E. coli coliform Selective Agar, Oxoid, UK) and Columbia Blood Agar (CBA) (EO Labs, UK) at $37^{\circ} \mathrm{C}$ overnight in $5 \% \mathrm{CO}_{2}$. Samples with low counts $(0-5 \mathrm{CFU})$ after $24 \mathrm{~h}$ growth were re-plated using $100 \mu \mathrm{l}$ of undiluted suspension. Rectal swabs were plated directly on agar as described above. To confirm presence of E. coli in a selection of stool samples, DNA was extracted directly from stool using Qiagen QIAamp DNA Stool Extraction Kit (Qiagen, UK) and gadA polymerase chain reaction (PCR) was performed as previously reported [17] (Additional file 1: Table S1). Samples were separately plated and all colonies frozen in glycerol bead stocks for future use.

\section{Sampling strategy}

To determine the optimum number of colonies to pick from patient samples, groups of $5,10,15$, and $20 \mathrm{E}$. coli single colonies were selected from 10 randomly selected samples. Donors were $E$. coli bacteraemia patients receiving antibiotics $(\mathrm{n}=5)$, and hospitalised patients who did not have bacteraemia and were not receiving antibiotics $(n=5)$. Individual colonies were boiled at $90{ }^{\circ} \mathrm{C}$ for 10 min to lyse the cells and release DNA; these were then subject to randomly-amplified-polymorphic-DNA (RAPD)-PCR using primers previously reported [18] (Additional file 1: Table S1). The number of different RAPD patterns obtained from $5,10,15$ or 20 single $E$. coli colonies was compared for each sample.

\section{Comparison of E. coli diversity between patient groups}

Single purple colonies from chromogenic agar identified as $E$. coli were randomly selected from different quadrants of the chromogenic agar plate, and sub-cultured on to LB agar (Oxoid, UK). Ten colonies per sample were subject to DNA extraction and RAPD-PCR as described above. The number of different RAPD patterns obtained was compared for each of the patient groups. Groups were compared pairwise by Mann-Whitney test; groups of more than two were compared by Kruskal-Wallis (Graph Pad Prism).

\section{Results and discussion Sampling strategy}

Although up to 10 distinct $E$. coli lineages can be present in the normal human enteric microbiota, medians of 2-3 types have been reported in patients in the community [6-10]. For a large clinical study in bacteraemia patients, that might include 100-200 hospitalised cases, exhaustive molecular analysis of 1000's of E. coli colonies would not be feasible. To determine a suitable number of $E$. coli colonies to analyse per patient when evaluating the diversity of $E$. coli lineages in the gut microbiota of hospitalized patients, we compared RAPD patterns of 20, 15,10 and $5 E$. coli colonies cultured from ten different in-patient faecal samples. We included equal numbers of samples from those diagnosed with bacteraemia and from those who had not been receiving any antibiotics for 30 days prior to sample collection. 
Examination of 15 colonies allowed detection of increased diversity in just one sample tested in each group compared with examination of 10 colonies. Further expansion to examine 20 colonies did not increase the observed diversity of $E$. coli per sample in either group. Taking all the data together, there was no difference in the number of potential different genotypes detectable by RAPD-PCR when selecting 10 or 20 colonies $(\mathrm{p}>0.05)$ (Fig. 1 and Additional file 1: Table S2). As such, for subsequent work, a practical approach of selecting 10 colonies per patient was adopted.

\section{Population of E. coli in different patient groups}

Enteric samples were collected from a larger cohort of 74 E. coli bacteraemia patients (receiving empiric antibiotics) and 70 control in-patients not receiving antibiotics. To control for the potential effect of antibiotic exposure, we included an additional group of 42 other Gram-negative (non $E$. coli) bacteraemia patients, who received the same empiric antibiotics as the $E$. coli bacteremia group. Samples were sought from all eligible patients during the study period and RAPD-PCR was undertaken in all cases where enteric samples yielded at least 10 colonies of viable $E$. coli on subculture (Additional file 1: Figure S1).

The median number of $E$. coli RAPD patterns recovered from 70 hospital in-patient controls, who were not receiving antibiotics, was two (range 1-5), in contrast to the 74 E. coli bacteraemia patients in whom a median of one RAPD type (range $1-4)$ was detected ( $p=0.029$ ). Despite being similarly antibiotic-treated, the median number of RAPD patterns detected in the 42 non- $E$. coli

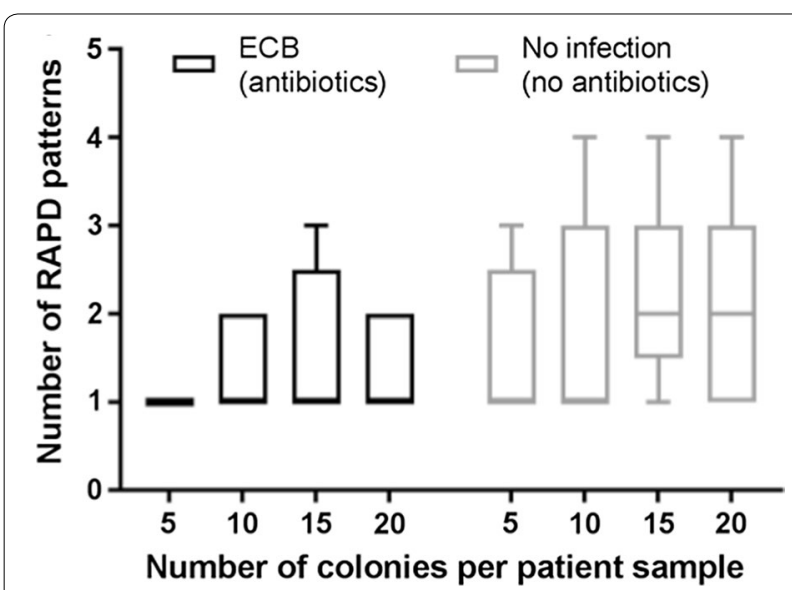

Fig. 1 Number of RAPD patterns detected when testing 5, 10, 15 or 20 E. coli colonies per patient sample. Enteric samples were tested from E. coli bacteraemia (ECB) patients ( $n=5$, receiving antibiotics), and non-antibiotic-exposed inpatient controls ( $n=5$, no infection, not receiving antibiotics). Different numbers of colonies were evaluated, as indicated and the number of distinct RAPD patterns enumerated. Plot shows median and interquartile range
Gram negative bacteraemia samples analysed was two (range 1-5) although the differences between the three groups overall was not significant $(\mathrm{p}=0.06)$ (Fig. 2).

We considered whether the type of sample affected our results and so compared data obtained from rectal swabs and faecal samples; rectal swabs were performed largely for the purpose of screening for carriage of carbapenemresistant organisms using risk-based algorithms and could represent a marker of prior healthcare exposure. Importantly, however, there was no significant difference between the two types of sample (Additional file 1: Figure S2). Rectal swabs may be obtained earlier during each hospital admission than stool samples, reducing the effect of recent antibiotic exposure on the diversity detected. The value of using rectal swabs is a useful observation, since inpatients are often unable to provide a faecal sample, while rectal swabs are increasingly obtained as part of routine screening practice.

Overall, we found a narrower diversity of $E$. coli in $E$. coli bacteraemia patients compared to control hospitalised patients. Although our study was not sufficiently powered to directly compare the E. coli bacteraemia group with the 'other Gram negative bacteraemia' group, our data suggest that the reduced diversity of $E$. coli in the enteric microbiota of $E$. coli bacteraemia patients may be specific to this group, and not necessarily related to concurrent antimicrobial exposure. We speculate that pathogenic E. coli strains causing bacteraemia may outcompete and dominate the gut microbiota. Whether this is related to past antimicrobial consumption or other pressures affecting the enteric reservoir of in-patients will require further study using specifically selected populations of patients with known antimicrobial history.

\section{Limitations}

Our study had limitations, in that enteric samples were only available from around a quarter of eligible patients with bacteraemia, and, for practical reasons, efforts to culture E. coli from all patients did not include heat shock or novobiocin-enrichment which, in separate studies, we found did improve yield (not shown). However, the number of samples evaluated was high, and there is no evidence to suggest these limitations would have affected any one patient group more than another. The number of different $E$. coli lineages detected in the enteric microbiota of our control groups is similar to that reported by other investigators [8] although, to our knowledge, acutely unwell hospital inpatients have not been studied previously.

A practical decision was made to sample ten colonies per patient, a strategy that means uncommon variants (present at a level of $10 \%$ or less) in the enteric sample will be identified less frequently $(\sim 35 \%)$ [13]. In our pilot 


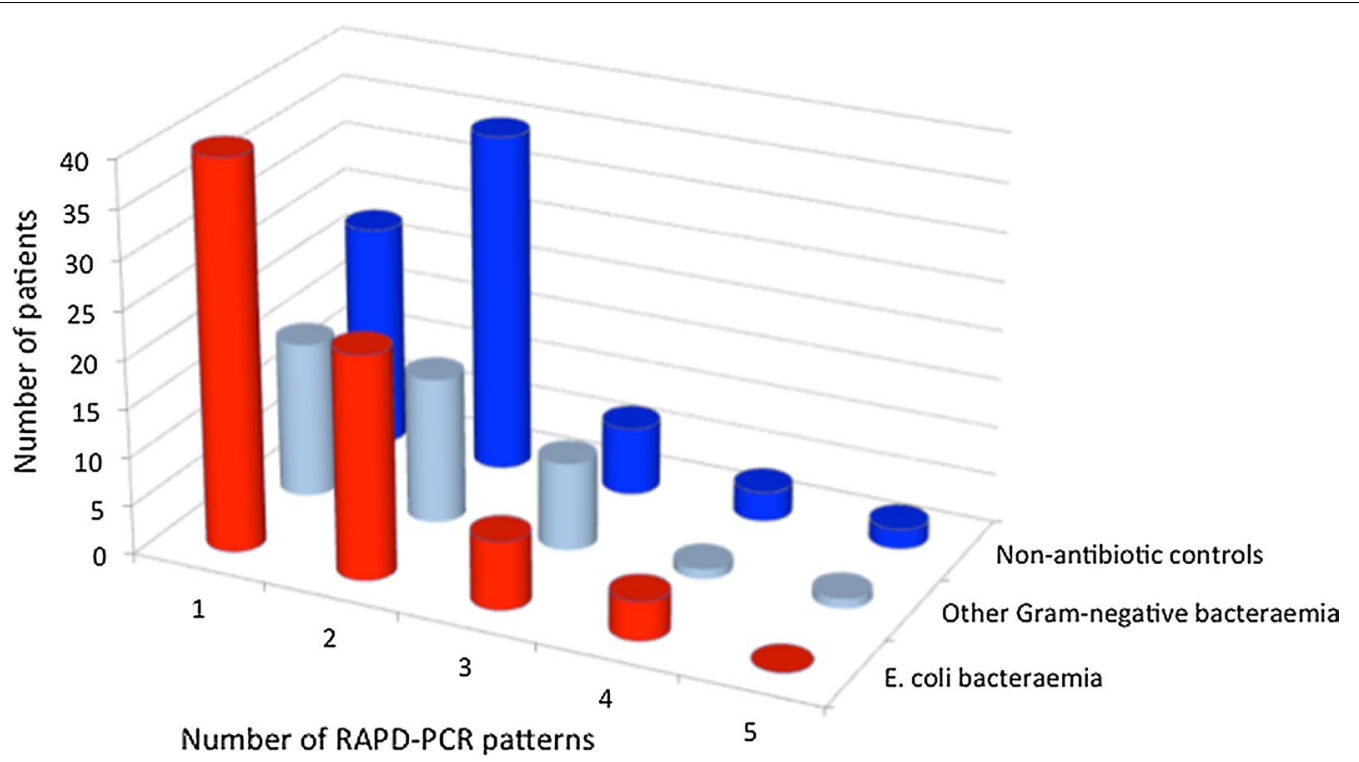

Fig. 2 Diversity of $E$. coli in enteric microbiota from patients with E. coli bacteraemia compared with other patient groups. Frequency of different numbers of RAPD patterns among E. coli bacteraemia patients $(n=74)$, patients with other Gram negative bacteraemia $(n=42)$, and non-antibiotic exposed inpatient controls who had no infection and were not taking antibiotics $(n=70)$. Although a difference was detected between the two main groups ( $E$. coli bacteraemia and non-antibiotic inpatient controls, $p=0.029$ ), there were no overall differences between the three groups $(p=0.06)$

study of hospitalised patients, increasing sampling from 10 to 20 colonies did not increase the yield of genetically distinct E. coli types. Notably this part of the study was markedly under-powered to detect very small differences such as those observed, and would have required many 100 's of samples to detect a clear difference; increasing colony sampling may have a greater effect in alternate non-hospitalised or healthy patient populations.

RAPD-PCR can reliably investigate the diversity of $E$. coli subclones in enteric microbiota [15] although cannot be used to finely discriminate between isolates at the genomic level; results may be refined using genomic DNA extraction methods. It does however provide an affordable and scalable method to determine the number of isolates to study using more expensive methods such as genome sequencing.

\section{Additional file}

Additional file 1. Sampling and diversity of Escherichia coli from the enteric microbiota in patients with Escherichia coli bacteraemia. Table S1. Primers used in this study. Table S2. Frequency of different RAPD patterns in each patient sample. Figure S1. Sample recruitment for study. Figure S2. E. coli diversity in rectal swab and stool samples.

\section{Abbreviations}

RAPD: randomly amplified polymorphic DNA; PCR: polymerase chain reaction; CFU: colony forming units; CBA: Columbia Blood Agar; NHS: National Health
Service; NIHR HPRU: National Institute for Health Research Health Protection Research Unit.

\section{Acknowledgements}

The authors thank the Imperial College NHS Trust Diagnostic Laboratory (Dr. Hugo Donaldson and colleagues and the BioAID team) for support and assistance with sample collection, and Dr. Kate Honeyford for advice on data analysis. SS acknowledges support of the NIHR Biomedical Research Centre awarded to Imperial College NHS Trust.

\section{Authors' contributions}

$M M, O B$, and IC were responsible for patients and sample recruitment; $M M, M E$ and $\mathrm{EJ}$ were responsible for sample analysis; $\mathrm{MM}$ and $\mathrm{EJ}$ were responsible for data analysis; NW and SS were responsible for project design and supervision; MM and SS wrote the first draft of the paper. All authors read and approved the final manuscript.

\section{Funding}

The research was funded by the National Institute for Health Research Health Protection Research Unit (NIHR HPRU) in Healthcare Associated Infections and Antimicrobial Resistance at Imperial College London in partnership with Public Health England (PHE). The views expressed are those of the authors and not necessarily those of the NHS, the NIHR, the Department of Health or Public Health England. The funder had no role in the design of the study or collection, analysis, and interpretation of data or in writing the manuscript.

\section{Availability of data and materials}

All data generated or analysed during this study are included in this published article (and its additional files).

\section{Ethics approval and consent to participate}

The collection and use of samples and consent process was approved by the Camden and Islington National Research Ethics Committee (Reference 14/ LO/2217).

\section{Consent for publication}

Not applicable. 


\section{Competing interests}

The authors declare they have no competing interests.

\section{Author details}

${ }^{1}$ Health Protection Research Unit in Healthcare Associated Infections and Antimicrobial Resistance, Department of Medicine, Imperial College Lon don, Hammersmith Campus, Du Cane Road, London W12 ONN, UK. ${ }^{2}$ Antimicrobial Resistance and Healthcare Associated Infections (AMRHAI) Reference Unit, National Infection Service, Public Health England, London, UK.

Received: 27 February 2019 Accepted: 5 June 2019

Published online: 13 June 2019

\section{References}

1. Schlackow I, Stoesser N, Walker AS, Crook DW, Peto TEA, Wyllie DH. Increasing incidence of Escherichia coli bacteraemia is driven by an increase in antibiotic-resistant isolates: electronic database study in Oxfordshire 1999-2011. J Antimicrob Chemother. 2012;67(6):1514-24.

2. Horner C, Fawley W, Morris K, Parnell P, Denton M, Wilcox M. Escherichia coli bacteraemia: 2 years of prospective regional surveillance (2010-12). J Antimicrob Chemother. 2014;69(1):91-100.

3. Abernethy JK, Johnson AP, Guy R, Hinton N, Sheridan EA, Hope RJ. Thirty day all-cause mortality in patients with Escherichia coli bacteraemia in England. Clin Microbiol Infect. 2015;21(3):251.e251-8.

4. Johnson JR, Johnston B, Clabots C, Kuskowski MA, Castanheira M. Escherichia coli sequence type ST131 as the major cause of serious multidrug-resistant E. coli infections in the United States. Clin Infect Dis. 2010;51(3):286-94.

5. Apperloo-Renkema HZ, Van der Waaij BD, Van der Waaij D. Determination of colonization resistance of the digestive tract by biotyping of Enterobacteriaceae. Epidemiol Infect. 1990;105(2):355-61.

6. Schlager TA, Hendley JO, Bell AL, Whittam TS. Clonal diversity of Escherichia coli colonizing stools and urinary tracts of young girls. Infect Immun. 2002;70(3):1225-9.

7. Chen SL, Wu M, Henderson JP, Hooton TM, Hibbing ME, Hultgren SJ, Gordon J. Genomic diversity and fitness of E. coli strains recovered from the intestinal and urinary tracts of women with recurrent urinary tract infection. Sci Transl Med. 2013:5(184):184ra160.

8. Nielsen KL, Dynesen P, Larsen P, Frimodt-Møller N. Faecal Escherichia coli from patients with E. coli urinary tract infection and healthy controls who have never had a urinary tract infection. J Med Microbiol. 2014;63(Pt 4):582-9.

9. Gordon DM, O'Brien CL, Pavli P. Escherichia coli diversity in the lower intestinal tract of humans. Environ Microbiol Rep. 2015:7(4):642-8.
10. Stoesser N, Sheppard AE, Moore CE, GolubchikT, Parry CM, Nget P, Saroeun M, Day NP, Giess A, Johnson JR, Peto TE, Crook DW, Walker AS, Walker and Modernizing Medical Microbiology Informatics Group. Extensive within-host diversity in fecally carried extended-spectrum beta-lactamase-producing Escherichia coli: implications for transmission analyses. J Clin Microbiol. 2015;53(7):2122-31.

11. Rhodes A, Evans LE, Alhazzani W, Levy MM, Antonelli M, Ferrer R, Kumar A, Sevransky JE, Sprung CL, Nunnally ME, Rochwerg B, Rubenfeld GD, Angus DC, Annane D, Beale RJ, Bellinghan GJ, Bernard GR, Chiche J-D, Coopersmith C, De Backer DP, French CJ, Fujishima S, Gerlach H, Hidalgo JL, Hollenberg SM, Jones AE, Karnad DR, Kleinpell RM, Koh Y, Lisboa TC, Machado FR, Marini JJ, Marshall JC, Mazuski JE, Mclntyre LA, McLean AS, Mehta S, Moreno RP, Myburgh J, Navalesi P, Nishida O, Osborn TM, Perner A, Plunkett CM, Ranieri M, Schorr CA, Seckel MA, Seymour CW, Shieh L, Shukri KA, Simpson SQ, Singer M, Thompson BT, Townsend SR, Van der Poll T, Vincent J-L, Wiersinga WJ, Zimmerman JL, Dellinger RP. Surviving sepsis campaign: international guidelines for management of sepsis and septic shock: 2016. Intensive Care Med. 2017:43(3):304-77.

12. Hibbing ME, Fuqua C, Parsek MR, Peterson SB. Bacterial competition: surviving and thriving in the microbial jungle. Nat Rev Microbiol. 2010;8(1):15-25.

13. Lautenbach E, Bilker WB, Tolomeo P, Maslow JN. Impact of diversity of colonizing strains on strategies for sampling Escherichia coli from fecal specimens. J Clin Microbiol. 2008;46(9):3094-6.

14. Maurer JJ, Lee MD, Lobsinger C, Brown T, Maier M, Thayer SG. Molecular typing of avian Escherichia coli isolates by random amplification of polymorphic DNA. Avian Dis. 1998;42(3):431-51.

15. Nielsen KL, Godfrey PA, Stegger M, Andersen PS, Feldgarden M, FrimodtMøller N. Selection of unique Escherichia coli clones by random amplified polymorphic DNA (RAPD): evaluation by whole genome sequencing. J Microbiol Methods. 2014;103:101-3.

16. Stephen AM, Cummings JH. The microbial contribution to human faecal mass. J Med Microbiol. 1980;13(1):45-56.

17. Doumith M, Day MJ, Hope R, Wain J, Woodford N. Improved multiplex PCR strategy for rapid assignment of the four major Escherichia coli phylogenetic groups. J Clin Microbiol. 2012;50(9):3108-10.

18. Radu S, Mutalib SA, Rusul G, Ahmad Z, Morigaki T, Asai N, Kim YB, Okuda J, Nishibuchi M. Detection of Escherichia coli O157:H7 in the beef marketed in Malaysia. Appl Environ Microbiol. 1998;64(3):1153-6.

\section{Publisher's Note}

Springer Nature remains neutral with regard to jurisdictional claims in published maps and institutional affiliations.
Ready to submit your research? Choose BMC and benefit from:

- fast, convenient online submission

- thorough peer review by experienced researchers in your field

- rapid publication on acceptance

- support for research data, including large and complex data types

- gold Open Access which fosters wider collaboration and increased citations

- maximum visibility for your research: over 100M website views per year

At BMC, research is always in progress.

Learn more biomedcentral.com/submissions 